\title{
High Proportion of Asymptomatic SARS-CoV-2 Infections in 9 Long- Term Care Facilities, Pasadena, California, USA, April 2020
}

\author{
Matt Feaster, Ying-Ying Goh
}

Our analysis of coronavirus disease prevalence in 9 long-term care facilities demonstrated a high proportion $(40.7 \%)$ of asymptomatic infections among residents and staff members. Infection control measures in congregate settings should include mass testing-based strategies in concert with symptom screening for greater effectiveness in preventing the spread of severe acute respiratory syndrome coronavirus 2 .


evere acute respiratory syndrome coronavirus 2 (SARS-CoV-2) is a novel human coronavirus that causes coronavirus disease (COVID-19). The disease was detected in the United States on January 20, 2020, and had caused $>1.7$ million cases and $>100,000$ deaths as of June 1, $2020(1,2)$. As the pandemic continues, data consistently show that older adults, particularly those with $\geq 1$ underlying medical conditions, experience higher hospitalization rates and increased vulnerability to in-hospital death $(3,4)$.

Long-term care facilities (LTCFs) in the United States, including skilled nursing facilities (SNFs) and assisted living facilities (ALFs), are populated by older adults and adults needing residential care for underlying medical conditions who are at increased risk of more severe COVID-19-associated illness $(4,5)$. ALF residents generally require a limited amount of care, such as help getting dressed or assistance with medications, whereas SNF residents have acute or chronic health conditions, or both, that require 24hour onsite medical care and often rehabilitative care and therapy.

The city of Pasadena, California, USA, is an independent local public health jurisdiction that has a

Author affiliation: City of Pasadena Public Health Department, Pasadena, CA, USA

DOI: https://doi.org/10.3201/eid2610.202694 disproportionately high representation of older adults compared with other southern California local public health jurisdictions. More than $12.0 \%$ of Pasadena's population is $>70$ years of age, compared with $9.1 \%$ in Los Angeles County (6), and Pasadena has $>1,253$ licensed SNF beds, which is 2.4 times the rate (per 100,000 residents) of SNF beds as in the Los Angeles County public health jurisdiction (7). The Pasadena Public Health Department (PPHD) recognized that this large population of medically fragile adults was at high risk for illness and death from COVID-19 as it spread through California, especially after early reports of nursing facility outbreaks in late February (8).

Extensive COVID-19-specific outreach and education efforts with skilled nursing facilities by PPHD staff began in late January 2020. In the second week of March, the PPHD received a report of laboratory-confirmed COVID-19 in a Pasadena resident (not facility-associated); a report of COVID-19 in a LTCF employee was received on March 31. By mid-April, the PPHD had opened investigations for facilities with $>1$ confirmed COVID-19 case in 14 of 15 SNFs in the city jurisdiction and 3 of 26 ALFs. By the end of April, 19 facilities in Pasadena had completed mandatory facilitywide screenings for SARS-CoV-2 to aid in the investigation and control of COVID-19 transmission.

\section{The Study}

Facilitywide testing of staff and residents was completed in all facilities by the end of April, with thousands of test results available by early May. This analysis was restricted to facilities with $\geq 3$ linked cases. Facilities excluded from the analysis had singular cases, non-epidemiology-linked cases, or no reported COVID-19 cases at the time of the initial mass testing. Of the 19 facilities, 9 (8SNFs and 1 ALF) had evidence 
of sustained transmission by investigation within the facility and were included in this analysis. Residents were included if they were listed on the facility's census sheet on the day the investigation was opened. All types of staff, both clinical and nonclinical, were required to participate.

A case-patient was defined as a person with a nasopharyngeal swab specimen that tested positive for SARS-CoV-2 by real-time reverse transcription PCR (rRT-PCR) at a commercial laboratory or the Los Angeles County Public Health Laboratory (Downey, CA, USA). Laboratory results were combined with case investigation data collected by PPHD public health nurses. Symptom data were extracted from case reports compiled during the case investigation (10), patient medical records (hospital and physician notes), and facility clinical staff assessments and records for residents. Residents and staff were classified as symptomatic if they had had $\geq 1$ new or worsened signs or symptoms of COVID-19 in the 14 days before nasal swab specimen collection. Persons with subjective fever or temperature $>100.0^{\circ} \mathrm{F}\left(37.8^{\circ} \mathrm{C}\right)$, muscle aches, cough, shortness of breath, fatigue, headache, new loss of taste or smell, sore throat, runny nose, nausea or vomiting, diarrhea, low oxygen saturation, or clinical oxygen requirement (as determined by the patient's physician) were classified as symptomatic (11).
A total of 1,093 persons (608 residents and 485 staff members) were eligible for rRT-PCR testing for SARSCoV-2 based on facilitywide testing strategies at the 9 LTCF sites (Table 1). Test results for 85.9\% (938/1,093) of the staff and residents were obtained by PPHD, specifically $95.7 \%(582 / 608)$ of residents and $73.6 \%$ (356/485) of staff. The overall population (residents and staff) prevalence of SARS-CoV-2 among these 9 facilities was $67.3 \%$ (631/938). The overall prevalence of asymptomatic infection among those who tested positive was $40.7 \%(257 / 631)$. The prevalence of SARSCoV-2 infection among staff involved with direct patient care, such as certified nursing assistants (CNAs), licensed vocational nurses (LVNs), registered nurses (RNs), and other caregivers $(68.5 \%, 150 / 219)$ was higher than among those not providing direct patient care, such as activity, dietary, and maintenance workers $(48.1 \%, 25 / 52)$. A larger percentage of female staff $(62.5 \%)$ than male staff $(46.5 \%)$ functioned in clinical roles. The prevalence of SARS-CoV-2 infection among all residents was $70.1 \%$ (408/582); among female residents, the prevalence was $71.4 \%(237 / 332)$, and among male residents, it was $68.4 \%(171 / 250)$. Female residents had a higher rate of asymptomatic infection $(51.0 \%, 121 / 237)$ than male residents $(47.4 \%, 81 / 171)$.

Varying levels of SARS-CoV-2 prevalence were identified across facilities. The lowest levels were among residents and staff in facility E $(30.6 \%$ of

\begin{tabular}{|c|c|c|c|c|}
\hline Characteristic & Total eligible & Persons tested & Confirmed COVID-19† & Asymptomatic infection $†$ \\
\hline Staff & 485 & 356 (73.4) & $223(62.6)$ & $55(24.7)$ \\
\hline Age, y, mean (SD) & 443 & $41.8(13.3)$ & $42.8(12.7)$ & $39.8(14.2)$ \\
\hline \multicolumn{5}{|l|}{ Sex } \\
\hline $\mathrm{F}$ & 328 & 249 (75.9) & $170(68.3)$ & 39 (22.9) \\
\hline M & 157 & $107(68.2)$ & $53(49.5)$ & $16(30.2)$ \\
\hline \multicolumn{5}{|l|}{ Staff role } \\
\hline Activities & 15 & $9(60.0)$ & $4(44.4)$ & 0 \\
\hline Administration & 32 & $26(81.3)$ & $18(69.2)$ & $5(27.8)$ \\
\hline Dietary & 42 & $31(73.8)$ & $16(51.6)$ & $2(12.5)$ \\
\hline Housekeeping & 19 & $14(73.7)$ & $8(57.1)$ & $2(25.0)$ \\
\hline Maintenance & 14 & $12(85.7)$ & $5(41.7)$ & $4(20.0)$ \\
\hline CNA & 149 & 115 (77.2) & $78(67.8)$ & $20(25.6)$ \\
\hline LVN & 66 & $46(70.0)$ & 34 (73.9) & $5(14.7)$ \\
\hline RN & 34 & $23(67.6)$ & $14(60.9)$ & $3(21.4)$ \\
\hline Other caregiver $\ddagger$ & 49 & 35 (71.4) & $24(68.6)$ & $3(12.5)$ \\
\hline Other/unknown $\ddagger$ & 65 & 45 (69.2) & $22(48.9)$ & $11(50.0)$ \\
\hline Residents & 608 & $582(95.7)$ & $408(70.1)$ & $202(49.5)$ \\
\hline Age, y, mean (SD) & 603 & $78.0(13.3)$ & $78.4(13.0)$ & $77.1(13.0)$ \\
\hline \multicolumn{5}{|l|}{ Sex } \\
\hline $\mathrm{F}$ & 347 & 332 (95.7) & $237(71.4)$ & $121(51.0)$ \\
\hline$M$ & 261 & $250(95.8)$ & $171(68.4)$ & $81(47.4)$ \\
\hline
\end{tabular}

*Values are no. (\%) except as indicated. CNA, certified nursing assistant; COVID-19, coronavirus disease; LVN, licensed vocational nurse; RN, registered nurse.

†Severe acute respiratory syndrome coronavirus 2 detected on nasopharyngeal swab tested by reverse transcription PCR. Asymptomatic infection includes confirmed COVID-19 cases with no reported typical or atypical symptoms of COVID-19. Percentage with results is equal to the number of persons with laboratory results by the number eligible in the facility. The percentage confirmed includes the number of persons with a positive PCR COVID-19 result by the number of persons with laboratory results.

‡Other caregivers include physical therapists, respiratory therapists, rehabilitation workers, and caseworkers. Others include web developers and marketing personnel. 
Table 2. Results from facilitywide testing by facility and association to the facility, Pasadena, California, USA, April 2020*

\begin{tabular}{|c|c|c|c|c|}
\hline Category & Total eligible, no. & Persons tested, no. (\%) & Confirmed COVID-19, no. (\%) & Asymptomatic infection, no. (\%) \\
\hline Total & 1,092 & $938(85.9)$ & $631(67.3)$ & $257(40.7)$ \\
\hline Staff & 485 & $356(73.6)$ & $223(62.6)$ & $55(24.7)$ \\
\hline Residents & 608 & $582(95.7)$ & $408(70.1)$ & $202(49.5)$ \\
\hline Facility A & 196 & $174(88.8)$ & $123(70.7)$ & $79(64.2)$ \\
\hline Staff & 109 & 88 (80.7) & $46(52.3)$ & $13(28.3)$ \\
\hline Residents & 87 & $86(98.9)$ & $77(89.5)$ & $66(85.7)$ \\
\hline Facility B & 87 & $86(98.9)$ & $69(80.2)$ & $27(39.1)$ \\
\hline Staff & 35 & $34(97.1)$ & $23(67.6)$ & $4(17.4)$ \\
\hline Residents & 52 & $52(100)$ & $46(88.5)$ & $23(50.0)$ \\
\hline Facility C & 112 & $109(97.3)$ & $90(82.3)$ & $34(37.8)$ \\
\hline Staff & 35 & $35(100)$ & $25(71.4)$ & $6(24.0)$ \\
\hline Residents & 77 & $74(96.1)$ & $65(87.8)$ & $28(43.1)$ \\
\hline Facility D & 134 & $122(91.0)$ & $88(72.1)$ & $35(39.8)$ \\
\hline Staff & 33 & $26(78.8)$ & $26(100)$ & $6(23.1)$ \\
\hline Residents & 101 & 96 (95.0) & 62 (64.6) & $29(46.8)$ \\
\hline Facility E & 98 & $71(72.4)$ & $18(25.4)$ & $9(50.0)$ \\
\hline Staff & 62 & 35 (56.5) & $7(20.0)^{\prime}$ & 2 (28.6) \\
\hline Residents & 36 & $36(100)$ & $11(30.6)$ & 7 (63.6) \\
\hline Facility $\mathrm{F}$ & 79 & $78(98.7)$ & 67 (85.9) & $14(20.9)$ \\
\hline Staff & 25 & $25(100)$ & $25(100)$ & $6(24.0)$ \\
\hline Residents & 54 & 53 (98.1) & $42(79.2)$ & $8(19.0)$ \\
\hline Facility G & 117 & $110(94.0)$ & $76(69.1)$ & $26(34.2)$ \\
\hline Staff & 21 & $16(76.2)$ & $16(100)$ & $3(18.8)$ \\
\hline Residents & 96 & $94(97.9)$ & $60(63.8)$ & $23(38.3)$ \\
\hline Facility $\mathrm{H}$ & 212 & $148(69.8)$ & $63(42.6)$ & $20(31.7)$ \\
\hline Staff & 142 & $87(61.3)$ & 36 (41.4) & $11(30.6)$ \\
\hline Residents & 70 & $61(87.1)$ & $27(44.3)$ & $9(33.3)$ \\
\hline Facility I & 57 & $50(87.7)$ & $37(74.0)$ & $13(35.1)$ \\
\hline Staff & 22 & 20 (90.9) & 19 (95.0) & $4(21.1)$ \\
\hline Residents & 35 & $30(85.7)$ & $18(60.0)$ & $9(50.0)$ \\
\hline \multicolumn{5}{|c|}{$\begin{array}{l}\text { *Results include the percentage of staff and residents who had a severe acute respiratory syndrome coronavirus } 2 \text { reverse transcription PCR test result } \\
\text { (positive or negative). A positive result was taken as confirmation of COVID-19 infection. Asymptomatic infection was defined as a confirmed COVID-19 } \\
\text { infection with no reported typical or atypical symptoms. Percentage of population with test results is equal to the number of persons with test results } \\
\text { divided by the number eligible staff/residents in the facility. Percentage of confirmed COVID-19 is equal to the number of persons with a positive test } \\
\text { result divided by the number of persons with test results. COVID-19, coronavirus disease. }\end{array}$} \\
\hline
\end{tabular}

residents [11/36], $20.0 \%$ of staff $[7 / 35])$, the highest among residents in facilities A $(89.5 \%, 77 / 86)$, B $(88.5 \%, 46 / 52)$, and C $(87.8 \%, 65 / 74)$ and among staff in facilities D (26/26), F (25/25), and G (16/16) (Table 2). The prevalence of asymptomatic infection among staff members ranged from $17.4 \%$ (facility B, 4/23) to $30.6 \%$ (facility $H, 11 / 36$ ) (Table 2). The prevalence of asymptomatic infection among residents ranged from $19.0 \%$ (facility $\mathrm{F}, 8 / 42$ ) to $85.7 \%$ (facility A, 66/77) (Table 2).

\section{Conclusions}

The ability of SARS-CoV-2 to spread rapidly among residents and staff in congregate settings poses a major infection control challenge. Our findings demonstrate a high proportion of asymptomatic infection, even within moderately affected facilities, and support the use of mass testing-based strategies in concert with symptom screening. Data from the facilitywide screenings indicate that the rate of asymptomatic infection among staff, on average, was 1 in 4 , and among residents was 1 in 2 .

Early in the COVID-19 pandemic, the supply of both nasopharyngeal swabs and test kits for
SARS-CoV-2 rRT-PCR testing in the United States was extremely limited and made available only for symptomatic persons meeting certain criteria determined by the Centers for Disease Control and Prevention (CDC) (12). Diagnostic testing remained limited for many weeks, and LTCFs relied on symptom screening to exclude potentially infectious staff from work. On March 30, CDC published a change for the COVID-19 period of exposure risk from onset of symptoms to 48 hours before symptom onset (13). This change meant that symptom screening alone could be insufficient in protecting LTCF residents from contracting COVID-19 from asymptomatic, but infectious, staff, and studies have suggested a role for asymptomatic transmission in COVID-19 outbreaks (14).

Our findings demonstrate a high prevalence of both symptomatic and asymptomatic COVID-19 infection among residents and staff in 9 LTCFs. Because the potential for asymptomatic transmission of SARS-CoV-2 is concerning, for greater effectiveness, infection control efforts in LTCFs should include both mass testing-based strategies and symptom screening. 


\section{About the Authors}

Dr. Feaster is the lead epidemiologist for the City of Pasadena Public Health Department, Pasadena, California, USA, and is responsible for communicable disease investigation and chronic disease health assessment for the city of Pasadena. Dr. Goh is the health officer and director for the City of Pasadena Public Health Department.

\section{References}

1. Centers for Disease Control and Prevention. Corona virus disease 2019 (COVID-19): cases in the U.S. [cited 2020 Jun 1]. https:/ / www.cdc.gov/coronavirus/2019-ncov/ cases-updates/cases-in-us.html

2. Harcourt J, Tamin A, Lu X, Kamili S, Sakthivel SK, Murray J, et al. Severe acute respiratory syndrome coronavirus 2 from patient with coronavirus disease, United States. Emerg Infect Dis. 2020;26:1266-73. https:/ / doi.org/ 10.3201/eid2606.200516

3. Garg S, Kim L, Whitaker M, O'Halloran A, Cummings C, Holstein R, et al. Hospitalization rates and characteristics of patients hospitalized with laboratory-confirmed coronavirus disease 2019-COVID-NET, 14 states, March 1-30, 2020. MMWR Morb Mortal Wkly Rep. 2020;69:458-64. https:/ / doi.org/10.15585/mmwr.mm6915e3

4. Zhao H, Huang Y, Huang Y. Mortality in older patients with COVID-19. J Am Geriatr Soc. 2020 May 25 [Epub ahead of print]. https://doi.org/10.1111/jgs.16649

5. Centers for Disease Control and Prevention. People who are at higher risk for severe illness [cited 2020 May 27]. https:/ / www.cdc.gov/coronavirus/2019-ncov/need-extraprecautions / people-at-higher-risk.html

6. US Census Bureau. Age and sex: table S0101 [cited 2020 May 27]. https:/ / data.census.gov
7. US Centers for Medicare and Medicaid Services. Nursing home compare; 2020 [cited 2020 May 28]. https:/ / www. medicare.gov/nursinghomecompare/search.html

8. Arons MM, Hatfield KM, Reddy SC, Kimball A, James A, Jacobs JR, et al.; Public Health - Seattle and King County and CDC COVID-19 Investigation Team. Presymptomatic SARS-CoV-2 infections and transmission in a skilled nursing facility. N Engl J Med. 2020;382:2081-90. https:/ / doi.org/10.1056/NEJMoa2008457

9. Centers for Disease Control and Prevention. CDC 2019-novel coronavirus (2019-NCoV) real-time RT-PCR diagnostic panel. 2020 [cited 2020 May 27]. https://www.fda.gov/ media/134922/download

10. California Department of Public Health. Communicable disease control forms [cited 2020 May 28]. https://www.cdph. ca.gov/Programs/PSB/Pages/CommunicableDisease Control.aspx

11. Centers for Disease Control and Prevention. Symptoms of coronavirus; 2020 [cited 2020 May 27]. https:/ / www.cdc.gov/ coronavirus/2019-ncov/symptoms-testing/symptoms.html

12. Centers for Disease Control and Prevention. Updated guidance on evaluating and testing persons for coronavirus disease 2019 (COVID-19) [cited 2020 May 27]. https://emergency.cdc.gov/han/2020/han00429.asp

13. Centers for Disease Control and Prevention. Public health recommendations for community-related exposure; 2020 [cited 2020 May 27]. https:/ / www.cdc.gov/coronavirus/ 2019-ncov/php/public-health-recommendations.html

14. Furukawa NW, Brooks JT, Sobel J. Evidence supporting transmission of severe acute respiratory syndrome coronavirus 2 while presymptomatic or asymptomatic. Emerg Infect Dis. 2020 Jun 21 [Epub ahead of print]. https://doi.org/10.3201/eid2607.201595

Address for correspondence: Matt Feaster, Pasadena Public Health Department, 1845 N Fair Oaks Ave, Pasadena, CA 91103, USA; email: mfeaster@cityofpasadena.net 